\title{
Bundesarbeitsgemeinschaft Mädchenpolitik e.V. gegründet!
}

Am 9.11.1999 haben Vertreterinnen verschiedener Landesarbeitsgemeinschaften Mädchenarbeit/Mädchenpolitik und andere jeweils landesweit agierende Trägerinnen von Mädchenarbeit in Dresden die erste bundesweite trägerübergreifende Vernetzung als Lobby für Mädchen gegründet.

Ziele der Bundesarbeitsgemeinschaft (BAG) sind:

- gemeinsame mädchenpolitische Interessen auf Bundesebene zu vertreten,

- ein Forum für die fachliche Weiterentwicklung der feministischen Arbeit mit Mädchen und jungen Frauen zu schaffen

- die Kooperation und Vernetzung der Mitglieder zu unterstützen.

Zum Beitritt und zur Mitarbeit sind eingeladen:

- Landesarbeitsgemeinschaften und landesweite Zusammenschlüsse der Mädchenarbeit

- Delegierte von Verbänden/Organisationen, die auf Bundesebene Ziele und Zwecke des Vereins unterstützen

- Delegierte von Institutionen und Expertinnen der Mädchenarbeit, deren Aufnahme durch die jeweilige Landesarbeitsgemeinschaft/den landesweiten Zusammenschluß der Mädchenarbeit/-politik empfohlen wird.

Sitz der neuen BAG ist Berlin.

Im Vorstand sind:

LAG »Mädchen und junge Frauen in Sachsen « e.V.: Brigitte Drechsel

LAG-Mädchenarbeit Hessen: Kirsten Langmaack

LAG-Mädchenarbeit in NRW e.V.: Ulrike Graff

Überbezirklicher Arbeitskreis feministische Mädchenarbeit (AKÜ) Hamburg:

Marja Evers Life e.V. Berlin: Rita Eichelkraut

Koordinationsstelle außerschulische Mädchenarbeit, AWO LV Brandenburg:

Ulrike Häfner, Claudia Wallner, Fortbildnerin Münster.

Kontaktadresse:

LAG »Mädchen und junge Frauen in Sachsen « e.V.

Brigitte Drechsel

Altenzeller Str. 19

01069 Dresden

fon + fax 0351.4716429 